\title{
The role of latitude, ultraviolet radiation exposure and vitamin D in childhood asthma and hayfever: an Australian multicenter study
}

Hughes AM, Lucas RM, Ponsonby A-L, Chapman C, Coulthard A, Dear K, Dwyer T, Kilpatrick TJ, McMichael AJ, Pender MP, Taylor BV, Valery P, van der Mei IAF, Williams D. The role of latitude, ultraviolet radiation exposure and vitamin $\mathrm{D}$ in childhood asthma and hayfever: an Australian multicenter study.

Pediatr Allergy Immunol 2010.

(c) 2010 John Wiley \& Sons A/S

Observations of increasing allergy prevalence with decreasing distance from the Equator and positive associations with ambient ultraviolet radiation have contributed to a growing interest in the possible role of vitamin $\mathrm{D}$ in the etiology of allergy. The aims of this study were to describe any latitudinal variation in the prevalence of childhood allergy in Australia and to evaluate, in parallel, the individual associations between ultraviolet radiation (UVR)- and vitamin D-related measures and hayfever asthma and both conditions. Participants were population-based controls who took part in a multicenter case-control study, aged 18-61 yr and resident in one of four study regions ranging in latitude from $27^{\circ} \mathrm{S}$ to $43^{\circ} \mathrm{S}$. Data were derived from a self-administered questionnaire, interview and examination by a research officer and biologic sampling. Latitude and longitude coordinates were geocoded from participants' residential locations and climatic data were linked to postcodes of current residence. Stored serum was analyzed for 25hydroxyvitamin D concentrations and silicone rubber casts of the skin were used as an objective measure of cumulative actinic damage. There was an inverse latitude gradient for asthma (a $9 \%$ decrease per

increasing degree of latitude); however, this pattern did not persist after adjusting for average daily temperature. There was no association between any of the UVR- or vitamin D-related measures and childhood asthma, but greater time in the sun in winter between the ages 6-15 yr was associated with an increase in the odds of having hayfever [adjusted odds ratios (OR) 1.29; 95\% CI 1.01-1.63]. Oral supplementation with cod liver oil in childhood increased the odds of a history of having both asthma and hayfever $(2.87 ; 1.00-8.32)$. Further investigation of the possible role of early vitamin D supplementation in the development of allergy is warranted. Our results also suggest that solar exposure during childhood may be important in allergic sensitization. Plausible explanations, including biologic mechanisms, exist for both observations.

\section{Ann Maree Hughes', Robyn Marjorie Lucas ${ }^{1}$, Anne-Louise Ponsonby', Caron Chapman ${ }^{3}$, Alan Coulthard ${ }^{4}$, Keith Dear', Terry Dwyer ${ }^{2}$, Trevor J. Kilpatrick $^{5}$, Anthony J. McMichael', Michael P. Pender ${ }^{4}$, Bruce V. Taylor ${ }^{6}$, Patricia Valery ${ }^{7}$, Ingrid A. F. van der $M i^{8}$ and David Williams ${ }^{9}$}

${ }^{1}$ National Centre for Epidemiology and Population Health, The Australian National University, Canberra, Australia, ${ }^{2}$ Murdoch Childrens Research Institute, Melbourne, Australia, ${ }^{3}$ Barwon Health, Geelong, Australia, ${ }^{4}$ The University of Queensland and the Royal Brisbane and Women's Hospital, Brisbane, Australia, ${ }^{5}$ Centre for Neuroscience, The University of Melbourne, Melbourne, Australia, ${ }^{6}$ Otago University, Christchurch, New Zealand, ${ }^{7}$ Queensland Institute of Medical Research, Brisbane, Australia, ${ }^{8}$ Menzies Research Institute, Hobart, Australia, ${ }^{9} \mathrm{~J}$ ohn Hunter Hospital, Newcastle, Australia

Key words: epidemiology; allergy; asthma; hayfever; allergic rhinitis; Australia; latitude; ultraviolet radiation; vitamin $\mathrm{D}$; food allergy; eczema

Ann Maree Hughes, National Centre for Epidemiology and Population Health, Building $62 \mathrm{cnr}$ Mills and Eggleston Rds, The Australian National University, Canberra 0200 Australia.

Tel.: +612 61252852

Fax: +61261250740

E-mail: annmaree.hughes@anu.edu.au

Accepted 23 July 2010
Positive associations between latitude and incidence and prevalence of multiple sclerosis $(1,2)$ and type 1 diabetes $(3,4)$ led to the proposal that ambient ultraviolet radiation (UVR) may be important to the development of other immune system-mediated disorders, including allergies. In Australia, a good location to explore geographic variation in disease frequency due to its broad range of latitude, universal healthcare system and relative genetic and ethnic homogeneity, 
rates of EpiPen prescriptions and hospital admissions for anaphylaxis (the most extreme allergic reaction) are more common in higher latitude, less sunny, southern regions (5). However, asthma prevalence has been shown to correlate negatively with latitude (lower prevalence at higher latitude) and positively with ambient UVR (4).

Underpinning these ecological studies is a growing interest in the possible role of vitamin $\mathrm{D}$ in the etiology of allergy (6-14). However, in the absence of information about demographics and environmental exposures at the individual level, it is not possible to assess whether the geographic patterns observed indicate a true effect of UVR or the role of confounders that may also vary by latitude. Thus, multicenter analytic epidemiologic studies are required.

The primary aims of these analyses were to further delineate any latitude variation in the prevalence of childhood allergy in Australia and to investigate the association between personal sun exposure and vitamin D-related measures and history of asthma and hayfever with onset before age 15. Based on the existing literature (4), our a priori hypotheses were that high latitude (low ambient UVR), based on residence at birth and at interview, would be associated with a lower frequency of allergy, while measures of higher personal UVR exposure and higher vitamin D status would be associated with increased odds of having a history of hayfever, asthma or both conditions in childhood.

\section{Methods}

Study design

The study population is composed of control participants from the Ausimmune Study ( $\mathrm{n}=$ $558 ; 60 \%$ of those invited and eligible to participate), a multicenter case-control study designed to examine environmental risk factors for a first clinical diagnosis of central nervous system demyelination (FCD). Control participants were randomly selected from the Australian Electoral Roll (compulsory registration for citizens $\geq 18 \mathrm{yr}$ ) within each study region (Brisbane $27^{\circ} \mathrm{S}$; Newcastle city and surrounds $33^{\circ} \mathrm{S}$; Geelong city and the western districts of Victoria $37^{\circ} \mathrm{S}$; and the island of Tasmania $43^{\circ} \mathrm{S}$ ) and did not have multiple sclerosis. They were matched to cases on age (within $2 \mathrm{yr}$ ) and sex; thus, they were aged between 18 and $61 \mathrm{yr}$ and predominantly female (as expected for FCD cases). A self-administered questionnaire and a face-to-face interview/examination by a research officer provided data on demographic characteristics, health history, environmental exposures, family history and potential confounders. The study was approved by nine regional Human Research Ethics Committees and written informed consent was obtained from all participants.

\section{Outcome and exposure}

The allergic end-points included the following: asthma (a history of ever having had doctordiagnosed asthma); hayfever (a problem with sneezing, runny or blocked nose without a cold or flu, sometimes accompanied by watery, itchy eyes); eczema (any itchy rash that was coming or going for at least 6 months and affected the folds of the elbows, behind the knees, in front of the ankles, under the buttocks or around the neck, ear or eyes), and food allergy (an allergic reaction to food that involved skin rash or hives or swelling of the mouth or throat or difficulty breathing). Complete information on allergy status, including age of onset, was available for $527(94.5 \%)$ controls. We differentiated between those who answered 'No' to each of the four questions about allergies ('no allergy'; $\mathrm{n}=213$ ) and those who reported a history of hayfever $(\mathrm{n}=63)$, asthma $(\mathrm{n}=32)$, both hayfever and asthma $(n=32)$ and other allergies $(n=47$; comprising eczema and/or food allergies) with onset before age 15 . Allergies with onset after age $14(\mathrm{n}=140)$ were censored. To maximize statistical power, all available controls with no reported allergies prior to age 15 were used as the reference group for these analyses. The results of separate analysis conducted using only the "no allergy' group as the reference category were of similar magnitude and in the same direction; these results are available upon request.

Family history of allergy was defined as a history of asthma or hayfever in at least one firstdegree relative (parent or sibling). Information about birth order and family structure, maternal breastfeeding, attendance at childcare before age 6 , pet ownership before age 1 and receipt of vitamin or mineral supplements up to age 15 [specifically, cod liver oil supplements (yes/no) and vitamin D supplements (yes/no)] were obtained by self-administered questionnaires, but numbers of participants reporting the latter were too few $(\mathrm{n}=8 ; 1.5 \%)$ to examine in detail. A research officer recorded details about natural skin color (in relation to photographic images), skin reaction to $1 \mathrm{~h}$ of summer sun, history of skin cancer (of which the majority will be basal cell carcinomas and, therefore, reflect both age and childhood sun exposure), history of 
tonsillectomy and/or adenoidectomy before age 15 , and body mass index (BMI).

Lifetime personal work and residence calendars were used by participants to record every location they had lived. Postcodes were assigned to Australian residential locations where sufficient detail of the location was provided. Postcodes and other geographic data (town, state, country) were then geocoded using ArcGIS (ArcGIS Software: Version 9.0; Environmental Systems Research Institute Inc., Redlands, CA, USA) to assign associated longitude and latitude coordinates. Meteorological data, including annual mean temperature $\left({ }^{\circ} \mathrm{C}\right)$, average relative humidity $(\%)$ and mean precipitation $(\mathrm{mm})$, population-weighted and aggregated at the level of postcode, were obtained from the Australian National Climate Centre of the Bureau of Meteorology Research Centre. Residential remoteness was used as a measure of exposure to a rural environment and determined by classifying Australian residential locations, using postcodes, according to the Australian Standard Geographical Classification (ASGC). The ASGC comprises five categories, each identifying noncontiguous regions as having a particular degree of remoteness ranging from 'Major cities of Australia' to 'Very Remote Australia'.

Original categories of self-reported hours per day in the sun on weekends and holidays between the ages of 6-10 and 11-15 yr (hereafter referred to as 'childhood time in sun') were re-coded as a continuous variable in hours, whereby: 'none' $=$ 0 ; '<1/2h' $=0.25$; '<1/2-1 h' $=0.75$; ' $1-2$ h' $=$ 1.5 ; ' $2-3$ h' $=2.5$; ' $3-4$ h' $=3.5$; '> 4 h' $=4.5$, and then added together, with separate variables for summer and winter. Using location data and satellite-derived estimates of average daily erythemally weighted ambient UVR (accessed from http://iridl.ldeo.columbia.edu/SOURCES/ .NASA/.GSFC/.TOMS/), we estimated, for each participant, average daily UVR for each month and each year for every location across the lifetime. These data were combined with information on hours outdoors from the lifetime calendar to calculate a personal leisure time UVR dose $\left(\mathrm{KJ} / \mathrm{m}^{2}\right)$ for every year of life for each participant (15) and then accumulated across the ages from 6 to 15 (hereafter referred as 'childhood UVR dose') and across the ages from age 6 to current age (hereafter referred as 'lifetime UVR dose') and modeled as a $\log$ variable. A leisure time (weekends and holidays) cumulative UVR dose was used rather than 'total sun exposure' (i.e., a combination of leisure and occupational exposure) because this has been found to have a higher correlation with actinic damage in an
Australian setting (15). Spectrophotometer readings of skin reflectance on the buttock (a body site unlikely to be exposed to sun) were converted to measures of cutaneous melanin density (15) and silicone rubber skin casts of the backs of both hands were scored on a scale from 1 to 6 (minimal to maximal actinic damage) and treated as an ordinal categorical variable; there were no actinic skin damage grades of 1 .

25-Hydroxyvitamin $D(25(\mathrm{OH})-\mathrm{D})$ was measured on stored serum (centrifuged, aliquoted and stored at $-0^{\circ} \mathrm{C}$ within $4 \mathrm{~h}$ of venipuncture) using liquid chromatography dual mass spectrometry. The 25(OH)-D level, available for 491 $(93 \%)$ participants, was de-seasonalized by fitting region-specific sine and cosine curves (16) to the individual data points.

\section{Statistical analysis}

Differences in the distribution of selected characteristics between the reference group and the allergy groups were tested with contingency table chi-square tests. Logistic multiple regression models and adjusted odds ratios (OR) with $95 \%$ confidence intervals $(95 \% \mathrm{CI})$ were used to determine the effects of demographic characteristics and different exposures on outcomes. All analyses were performed using Stata (StataCorp. Stata Statistical Software: Release 9.2; Stata Corporation, College Station, TX, USA).

\section{Results}

\section{General features}

Overall, the prevalence of childhood hayfever and/or asthma among Ausimmune Study controls was $24.1 \%(22.0 \%$ in men and $24.7 \%$ in women). Demographic characteristics of the study population are shown in Table 1 . There was a strong predominance of women $(77.6 \%$ vs. $22.4 \%$ ), reflecting the sex distribution of the Ausimmune Study cases, but participants with and without allergy were similar with respect to sex, age, and level of education. As expected, there was a strong association with history of allergy in first-degree family members and, therefore, all subsequent regression analyses were adjusted for this variable. The average age of childhood asthma onset (in years) was significantly younger than that for childhood hayfever (mean \pm standard deviation $=4.4 \pm 3.8$ and $8.3 \pm 4.3$, respectively; $\mathrm{p}<0.0001$ ). Skin color, skin reaction to sun, and buttock melanin density were not statistically related to any subtype of childhood allergy (data not shown). 
Table 1. Demographic characteristics of study subjects

\begin{tabular}{|c|c|c|c|c|c|}
\hline & Reference group* & Hayfever & Asthma & Hayfever and asthma & \\
\hline & $(n=353)$ & $(n=63)$ & $(n=32)$ & $(n=32)$ & \\
\hline & $\mathrm{n}(\%)$ & n (\%) & $\mathrm{n}(\%)$ & $n(\%)$ & $\mathrm{p}$-value \\
\hline \multicolumn{6}{|l|}{ Sex } \\
\hline Female & $272(77.1)$ & 52 (82.5) & $24(75.0)$ & $25(78.1)$ & \multirow[t]{2}{*}{0.78} \\
\hline Male & 81 (22.9) & $11(17.5)$ & 8 (25.0) & $7(21.9)$ & \\
\hline \multicolumn{6}{|c|}{ Age at interview (yr) } \\
\hline $18-29$ & $60(17.0)$ & $8(12.7)$ & $5(15.6)$ & $11(34.4)$ & \multirow[t]{4}{*}{0.55} \\
\hline $30-39$ & $123(34.8)$ & $23(36.5)$ & $11(34.4)$ & $8(25.0)$ & \\
\hline $40-49$ & $109(30.9)$ & $21(33.3)$ & $11(34.4)$ & $9(28.1)$ & \\
\hline $50-59$ & 161 (17.3) & $11(17.5)$ & $5(15.6)$ & $4(12.5)$ & \\
\hline \multicolumn{6}{|c|}{ Highest level of education } \\
\hline Up to $\mathrm{Yr} 10$ & $112(33.1)$ & 19 (31.7) & $10(32.3)$ & $8(27.6)$ & \multirow[t]{3}{*}{0.57} \\
\hline Yr12/TAFE $\dagger$ & $149(44.1)$ & $22(36.7)$ & $11(35.5)$ & $11(37.9)$ & \\
\hline Tertiary & $77(22.8)$ & 19 (31.7) & $10(32.3)$ & $10(34.5)$ & \\
\hline \multicolumn{6}{|c|}{ Family history of hayfever and/or asthma } \\
\hline No & $107(33.4)$ & $8(13.8)$ & $4(13.3)$ & $2(6.9)$ & \multirow[t]{2}{*}{$<0.0001$} \\
\hline Yes & $213(66.6)$ & 50 (86.2) & $26(86.7)$ & $27(93.1)$ & \\
\hline
\end{tabular}

*Reference group comprises participants with no reported allergies prior to age 15 .

$\uparrow$ Technical and Further Education.

\section{Latitude}

There was no association between latitude at birth and having a history of childhood asthma, but we observed a strong inverse relationship with latitude at interview. The latter corresponded to an overall $9 \%$ decrease in odds of childhood asthma per increasing degree of latitude (Table 2) and an $82 \%$ decrease in the odds within the highest latitude category compared to the lowest: ' $33^{\circ} \mathrm{S}$ ': adjusted OR $0.54 ; 95 \%$ CI $0.18-1.63$, ' $37^{\circ} \mathrm{S}$ ': $0.42 ; 0.16-1.12,{ }^{\circ} 43^{\circ} \mathrm{S}$ ': 0.18 ; 0.04-0.86 compared to the reference category of ' $27^{\circ} \mathrm{S}$ ' ( $\mathrm{p}$ for trend $=0.01$; Fig. 1). This effect, however, did not persist after further adjustment for average daily temperature ( $\mathrm{p}$ for trend $=$ 0.57), which was positively associated with childhood asthma (Table 2); whereas adjustment for lifetime UVR dose made no material difference to the estimates ( $p$ for trend $=0.03$ ). In contrast, there was no evidence of any latitudinal variation or consistent effect of temperature, relative humidity or precipitation influencing childhood history of hayfever or both hayfever and asthma (Table 2).

\section{UVR-related measures}

Childhood time in sun in winter was associated with increased odds of hayfever (Table 2). The size of the effect was of similar magnitude for childhood time in the sun in summer but this association did not reach statistical significance (Table 2). None of the UVR-related measures (childhood time in the sun in summer and winter, childhood and lifetime UVR dose or increasing sun exposure over the life course as measured by actinic skin damage score and history of skin cancer) were shown to be related to either asthma or both hayfever and asthma (Table 2). Further adjustment for skin phenotype using subjective (skin color, skin reaction to sun) and objective (cutaneous melanin density) measures did not alter these findings (data not shown).

\section{Vitamin D-related measures}

There was no statistically significant association between $25(\mathrm{OH})-\mathrm{D}$ levels at interview and childhood hayfever, asthma or both conditions (Table 2). Cod liver oil supplementation up to age 15 was associated with an increase in odds of having a history of both hayfever and asthma in childhood but was not significantly associated with asthma or hayfever on their own.

\section{Other allergies}

We also examined whether food allergy $(n=14)$ or eczema $(n=35)$ in childhood were associated with latitude or any of the UVR- or vitamin D-related measures. We did not observe any variation in latitude nor was there any evidence that any of the measures were associated with the development of either of these allergies in childhood (data not shown). 
UVR exposure and childhood allergy

Table 2. Associations between latitude, ultraviolet radiation (UVR)- and vitamin D-related measures and history of hayfever and asthma before age 15.

\begin{tabular}{|c|c|c|c|c|c|c|}
\hline & \multicolumn{2}{|c|}{ Hayfever ( $\mathrm{n}=63$ ) } & \multicolumn{2}{|c|}{ Asthma ( $\mathrm{n}=32)$} & \multicolumn{2}{|c|}{ Hayfever and asthma $(n=32)$} \\
\hline & OR $(95 \% \mathrm{Cl})^{*}$ & p-value & OR $(95 \% \mathrm{CI})^{*}$ & $\mathrm{p}$-value & OR $(95 \% \mathrm{Cl})^{*}$ & $\mathrm{p}$-value \\
\hline \multicolumn{7}{|l|}{ Meteorological data } \\
\hline Temperature & $0.99(0.91-1.08)$ & 0.86 & $1.17(1.03-1.34)$ & 0.01 & $1.04(0.92-1.18)$ & 0.53 \\
\hline Relative humidity & $0.97(0.89-1.06)$ & 0.50 & $0.90(0.80-1.01)$ & 0.08 & $0.93(0.83-1.05)$ & 0.26 \\
\hline Precipitation & $0.35(0.09-1.41)$ & 0.14 & $1.29(0.50-3.28)$ & 0.60 & $1.18(0.39-3.61)$ & 0.77 \\
\hline \multicolumn{7}{|l|}{ Latitude of residence } \\
\hline At birth & $1.00(0.99-1.01)$ & 0.88 & 1.01 (0.99-1.03) & 0.07 & $0.99(0.97-1.02)$ & 0.66 \\
\hline At interview & $1.00(0.96-1.06)$ & 0.72 & $0.91(0.84-0.98)$ & 0.01 & $0.97(0.91-1.05)$ & 0.46 \\
\hline \multicolumn{7}{|l|}{ UVR-related measures } \\
\hline Childhood time in sun - summer & $1.23(0.92-1.64)$ & 0.17 & $0.95(0.67-1.36)$ & 0.78 & $0.85(0.60-1.21)$ & 0.37 \\
\hline Childhood time in sun - winter & $1.29(1.01-1.63)$ & 0.04 & $0.87(0.64-1.18)$ & 0.37 & $1.03(0.74-1.43)$ & 0.87 \\
\hline Childhood UVR dose - summer & $1.34(0.57-3.11)$ & 0.50 & $1.44(0.46-4.58)$ & 0.53 & $0.69(0.26-1.88)$ & 0.47 \\
\hline Childhood UVR dose - winter & $0.98(0.66-1.45)$ & 0.92 & $1.13(0.68-1.92)$ & 0.62 & $1.14(0.64-2.05)$ & 0.65 \\
\hline Childhood UVR dose - total & $1.14(0.52-2.47)$ & 0.74 & $1.41(0.48-4.11)$ & 0.53 & $0.73(0.26-2.05)$ & 0.55 \\
\hline Lifetime UVR dose & $1.83(0.76-4.44)$ & 0.18 & $3.20(0.89-11.56)$ & 0.08 & $0.66(0.21-2.07)$ & 0.48 \\
\hline Actinic skin damage score $\dagger$ & $0.87(0.66-1.14)$ & 0.31 & $1.16(0.82-1.64)$ & 0.41 & $1.13(0.77-1.65)$ & 0.53 \\
\hline History of skin cancer & $0.42(0.09-1.86)$ & 0.25 & $0.87(0.19-4.02)$ & 0.86 & - & \\
\hline \multicolumn{7}{|l|}{ Vitamin D-related measures } \\
\hline 25(OH)-D level at interview $\ddagger$ & $1.06(0.85-1.33)$ & 0.59 & $0.83(0.62-1.12)$ & 0.22 & $1.33(0.95-1.86)$ & 0.10 \\
\hline Receipt of cod liver oil up to age 15 & $1.20(0.55-2.63)$ & 0.65 & $1.34(0.43-4.19)$ & 0.61 & $2.87(1.00-8.32)$ & 0.05 \\
\hline
\end{tabular}

Significant results (95\% Cls do not include 1) are bold.

*ORs are relative to the reference group, comprising participants with no reported allergy history prior to age $15(\mathrm{n}=353)$, and adjusted for age, sex, education, family history of hayfever and/or asthma.

†Additionally adjusted for left/right hand.

¥Additionally adjusted for body mass index.

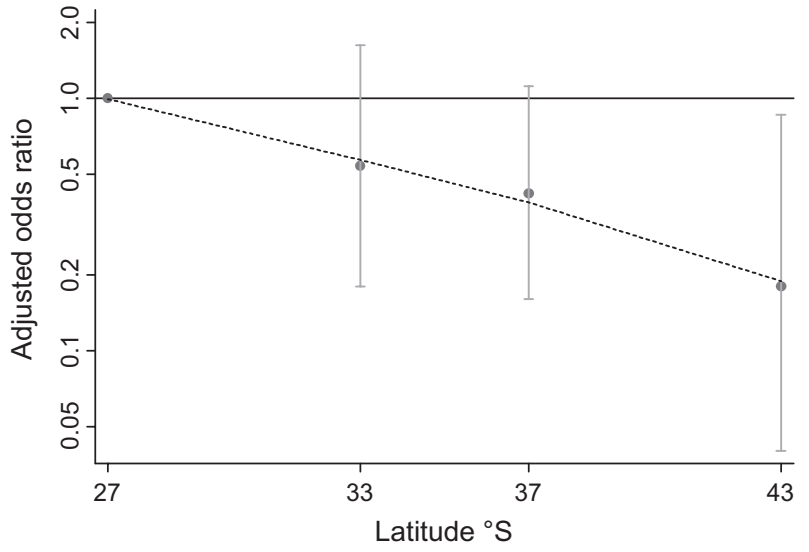

Fig. 1. Variation in childhood asthma by latitude in the Ausimmune Study controls. Data are odds ratios adjusted for age, sex, education, and family history of hayfever and/ or asthma with $95 \%$ confidence intervals in error bars.

\section{Additional analyses}

There was no association between either asthma, hayfever or both conditions and having been breastfed, daycare attendance, pet ownership, removal of tonsils and/or adenoids or living in a rural environment (data not shown). In general, measures of greater sibling exposure (birth order; cumulative exposure to siblings before age 2 and before age 6; sibship size; number of older and younger siblings) showed odds less than one of having an allergy in childhood, but none reached statistical significance (e.g., $4+$ siblings and hayfever: $0.16 ; 0.02-1.27)$. We found no evidence of effect modification of the sibling-allergy association by childhood time in the sun in summer or winter. Further, adjusting for sibling exposure made no material difference to the results reported here.

\section{Discussion}

This is the first epidemiological study to examine the association between UVR and history of allergy using both ecological data and direct measures of sun exposure and vitamin D supplementation in individuals. Our finding for odds of childhood asthma replicate previous Australian findings (4) and those from a worldwide meta-analysis of centers participating in the International Study of Asthma and Allergies in Childhood (ISAAC) (17) showing an inverse latitude gradient for asthma prevalence; however, in our data, we have shown that this pattern was accounted for by non-UVR regional climatic differences. Further, in a direct examination of self-reported personal time in the sun, cumulative UVR dose and vitamin D-related measures, we found no association with childhood asthma suggesting that these data do support an adverse 
effect of UVR for this condition. On the contrary, however, we observed an increase in odds of hayfever associated with childhood time in sun in winter and an increase in odds of having both hayfever and asthma with childhood supplementation of cod liver oil.

The main strength of this study lies in the broad range of information collected, which allowed for an examination of ecological factors as well as detailed measures of sun exposure and vitamin $\mathrm{D}$ status in relation to markers of allergy at the individual level. Moreover, it was possible to undertake a life course perspective and to control for important potential confounders including socioeconomic status and BMI (which may be related to higher vitamin $\mathrm{D}$ intake and lower vitamin D status, respectively), as well as factors related to the hygiene hypothesis. It might be argued that the present findings may not be fully generalizable to the general population because the participants included in the current analyses are not a random sample of the population but were matched to the Ausimmune Study cases. However, the participants should be representative of the sex and age group from which they were selected and, importantly, the prevalence of allergy in this sample is comparable with that reported in the Australian population (18). Recall bias may have affected the ascertainment of early life allergies and exposures and the self-reported, non-verified, nature of the questionnaire data lends itself to misclassification. We recognize that allergic factors play a role in many, but not every, case of asthma; however, we were unable to distinguish between allergic and non-allergic asthma in these data. Further, our measure of supplement use in childhood was crude and we lacked information about duration and compliance. However, standardized diseasespecific questions, rather than an open question that enquired about all chronic conditions, were included for each of the allergy end-points. In addition, lifetime calendars were employed to facilitate recall of past exposure to UVR at different periods of life.

Geographic latitude at places of residence is routinely used in ecological studies as a surrogate for UVR exposure because ambient UVR at Earth's surface varies inversely (approximately) with latitude (5). However, our findings suggest that the inverse association of childhood asthma with latitude is because of confounding by other climatic factors, rather than reflecting a direct association with UVR exposure. Specifically, the pattern we observed for asthma did not persist after adjusting for average daily temperature, implying that latitude might reflect regional variations in other ecological factors, such allergens, outdoor air pollution or infection, which have been linked to increased asthma symptoms (4). If true, this effect may be disease-specific, as hayfever, eczema, and food allergy were not related to climatic conditions in these data. Similarly, results from the ISAAC Study (17) show that climate may affect the prevalence of some allergies, but not others, in children. Latitude effects have also been attributed to differences in indoor humidity or altitude (17) and socioeconomic disparity (19), although the latter is not likely to be the explanation here. There are also suggestions that latitude might reflect genetic influences or cultural differences in child-rearing occurring within language borders (20). Alternatively, people diagnosed with asthma in childhood may simply move away from locations where conditions trigger asthma symptoms to climates more favorable to their disease.

No geographic variability was observed for a history of food allergy in childhood in these data. This is inconsistent with other Australian data (5). However, without information on anaphylaxis, we were unable to corroborate this pattern at an individual level.

Our observation of a trend of increasing odds of a history of hayfever in childhood with increasing time in the sun in winter is supportive of a positive association between early exposure to UVR/vitamin D and allergy in general. The apparent absence of an effect of UVR dose may indicate that 'time in the sun' measures more than erythemal UVR and reflects a different aspect of being outdoors. There is some evidence linking current vitamin D levels with an increase in prevalence of allergic rhinitis at all ages, with risk most prominent in the young (11), but this may represent confounding by children with higher vitamin $\mathrm{D}$ levels having greater exposure to outdoor allergens. For asthma, the reverse appears to be true, with reported associations between low vitamin D levels and childhood asthma severity (13) and poorer lung function in adult asthmatics $(7,14)$. However, exposure to higher maternal 25(OH)-vitamin D concentrations in late pregnancy may increase risk of asthma at age 9 (10) and recent evidence suggests that both very low and very high $25(\mathrm{OH})-\mathrm{D}$ concentrations may be associated with significant increases in total and specific IgE production (8).

Our findings of an association between cod liver oil supplementation and allergy are consistent with those from the Finnish birth cohort study (7), which reported OR of 1.35 (0.99-1.8) for asthma and $1.66(1.1-1.6)$ for allergic rhinitis 
by adulthood comparing regular vs. no vitamin D supplementation in the first year of life. Given that the participants in our study were adults recalling supplementation in their early lives some degree of recall error is likely, although the magnitude of the effect argues against this. The stronger association with supplementation seen in those with both hayfever and asthma, compared to those with either asthma or hayfever alone, may reflect more potent immunomodulatory effects of dietary vitamins or metabolic differences in children with greater hypersensitivity. Confounding by vitamin A or some unmeasured determinants could have contributed to the apparent effects of cod liver oil supplementation (6). It is also possible that the association between supplementation and allergic disease is because of reverse causation; that is, that early symptoms of allergy led to treatment with cod liver oil by concerned parents.

The findings reported here add to previous reports demonstrating a pro-allergic effect of oral vitamin $\mathrm{D}$ supplementation in a variety of forms (21). While the results from prenatal dietary studies $(9,12)$ showing a protective effect of vitamin $\mathrm{D}$ against allergies in offspring appear to be in contrast to our findings, these could be because of factors related to overall healthier maternal dietary patterns or lifestyle. Prenatal mechanisms might also underlie the observed effects; for example, responses to vitamin D exposure may vary between naïve and more mature T-cells (22). Alternatively, the effects of exposure to vitamin supplements on development of immunologically-mediated diseases may be different during the early postnatal period, when the immune system is 'learning' how to respond to new environmental stimuli, than at later times of life (23). In the treatment of asthmatic children, vitamin $\mathrm{D}_{3}$ may reduce incidence of attacks compared to placebo (24) but shows no beneficial adjuvant effect in a low ambient UV setting (25). More clinical studies are needed to determine whether such findings differ in high UV environments, such as Australia. The mechanisms by which vitamin $\mathrm{D}$ may affect manifestation of allergy are also unclear but may involve a blocking of $\mathrm{T}_{\mathrm{H}} 1$-mediated immune activity and a shift in T-cell responses toward $\mathrm{T}_{\mathrm{H}} 2$ phenotypes (26). Mice given supplements of vitamin $\mathrm{D}$ in early life show a sustained proliferation of allergy-inducing $\mathrm{T}_{\mathrm{H}} 2$ cells (27) and vitamin D-receptor knockout mice fail to develop allergic asthma (28). A number of vitamin D-regulated genes have also been linked to risk of atopy and asthma $(29,30)$.

\section{Conclusion}

In this analysis, we have shown that the inverse association between latitude and asthma does not reflect UVR, but is accounted for by other climatic factors such as temperature. We report data in which supplementation with cod liver oil before age 15 is associated with increased odds of having both asthma and hayfever, providing a rationale for an evaluation of the possible role of early vitamin D supplementation in the development of childhood allergy. That patterns of solar exposure during childhood may also be important in allergic sensitization, based on direct measures of sun exposure in individuals, is new and argues against the hypothesis that the influence of vitamin $\mathrm{D}$ on allergy is confined to its supplementation role (21). Our observations, based on both UVR and vitamin D, also now highlight the possibility that mode of delivery of vitamin D (supplementation with oral doses of derived vitamin $\mathrm{D}$ vs. vitamin $\mathrm{D}$ production in the skin following sunlight exposure) may result in similar patterns and further data on the possibly related role for UVR exposure is required.

\section{Acknowledgments}

We acknowledge the outstanding contribution to the Ausimmune Study of the research nurses who undertook all data collection: Susan Agland, Barbara Alexander, Zoe Dunlop, Anne Wright, Rosalie Scott, Jannie Selvidge, Marie Steele, Katherine Turner, Brenda Wood and the study project officers, Jane Gresham, Helen Rodgers, and Camilla Jozwick. We also thank $\mathrm{Mr}$ Ivan Hanigan for assistance with geocoding participants' residential histories. Dr Hughes is supported by an Australian NHMRC Postdoctoral Research Fellowship (Grant \#358672).

\section{References}

1. Miller DH, Hammond SR, Mcleod JG, Purdie G, SkeGG DC. Multiple sclerosis in Australia and New Zealand: are the determinants genetic or environmental? J Neurol Neurosurg Psychiatry 1990: 53: 903-5.

2. TAYLOR BV, LuCAS RM, DeAR K, et al. Latitudinal variation in incidence and type of first central nervous system demyelinating events. Mult Scler 2010: 16: 398 405.

3. DERIG. Geographic patterns of childhood insulindependent diabetes mellitus. Diabetes Epidemiology Research International Group. Diabetes 1988; 37: 1113 9.

4. Staples JA, Ponsonby Al, Lim Ll, McMichael AJ. Ecologic analysis of some immune-related disorders, including type 1 diabetes, in Australia: latitude, regional ultraviolet radiation, and disease prevalence. Environ Health Perspect 2003: 111: 518-23.

5. Mullins RC, Clark S, Camargo CA. Regional variation in epinephrine autoinjector prescriptions in Australia: more evidence for the vitamin D-anaphylaxis hypothesis. Ann Allergy Asthma Immunol 2009: 103: 488-95. 


\section{Hughes et al.}

6. Hyppönen E, Sovio U, Wust M, et al. Infant vitamin d supplementation and allergic conditions in adulthood: northern Finland birth cohort 1966. Ann N Y Acad Sci 2004: 1037: 84-95.

7. Black PN, Scragg R. Relationship between serum 25hydroxyvitamin $\mathrm{d}$ and pulmonary function in the third national health and nutrition examination survey. Chest 2005: 128: 3792-8.

8. Hyppönen E, Berry DJ, Wust M, Power C. Serum 25hydroxyvitamin D and $\operatorname{IgE}$ - a significant but nonlinear relationship. Allergy 2009: 64: 613-20.

9. Devereux G, Litonjua AA, Turner SW, et al. Maternal vitamin D intake during pregnancy and early childhood wheezing. Am J Clin Nutr 2007: 85: 853-9.

10. Gale CR, Robinson SM, Harvey NC, et al. Maternal vitamin D status during pregnancy and child outcomes. Eur J Clin Nutr 2008: 62: 68-77.

11. Wust M, Hyppönen E. Vitamin D serum levels and allergic rhinitis. Allergy 2007: 62: 1085-6.

12. Erkkola M, Kaila M, Nwaru BI, et al. Maternal vitamin $\mathrm{D}$ intake during pregnancy is inversely associated with asthma and allergic rhinitis in 5-year-old children. Clin Exp Allergy 2009: 39: 875-82.

13. Brehm JM, Celedon JC, Soto-Quiros ME, et al. Serum vitamin D levels and markers of severity of childhood asthma in Costa Rica. Am J Respir Crit Care Med 2009: 179: 765-71.

14. Sutherland ER, Goleva E, Jackson LP, Stevens AD, LEUNG DY. Vitamin D levels, lung function and steroid response in adult asthma. Am J Respir Crit Care Med 2010: 181: 699-704.

15. Lucas RM, Ponsonby AL, Dear K, et al. Associations between silicone skin cast score, cumulative sun exposure, and other factors in the Ausimmune Study: a multicenter Australian study. Cancer Epidemiol Biomarkers Prev 2009: 18: 2887-94.

16. Halberg F. Chronobiology. Annu Rev Physiol 1969: 31: $675-725$.

17. Weiland SK, Husing A, Strachan DP, Rzehak P, PEARCE N. Climate and the prevalence of symptoms of asthma, allergic rhinitis, and atopic eczema in children. Occup Environ Med 2004: 61: 609-15.

18. Australian Centre for Asthma Monitoring. Asthma in Australia 2008. AIHW Asthma Series no 3. Canberra: AIHW, 2008: 1-248.
19. Franco JM, Gurgel R, Sole D, Lucia Franca V, Brabin B. Socio-environmental conditions and geographical variability of asthma prevalence in Northeast Brazil. Allergol Immunopathol (Madr) 2009: 37: 11621.

20. Wust M, Dharmage S, Andre E, et al. Latitude, birth date, and allergy. PLoS Med 2005: 2: e294.

21. Wust M. Allergy risk of vitamin D supplements has been described in various settings. J Allergy Clin Immunol 2008: 121: 1065-6; author reply 66.

22. Annesi-Maesano I. Perinatal events, vitamin D, and the development of allergy. Pediatr Res 2002: 52: 3-5.

23. Holt PG, Jones CA. The development of the immune system during pregnancy and early life. Allergy 2000: 55: 688-97.

24. Urashima M, Segawa T, Okazaki M, Kurihara M, WADA Y, IDA H. Randomized trial of vitamin D supplementation to prevent seasonal influenza A in schoolchildren. Am J Clin Nutr 2010: 91: 125560 .

25. Majak P, Rychlik B, Stelmach I. The effect of oral steroids with and without vitamin D3 on early efficacy of immunotherapy in asthmatic children. Clin Exp Allergy 2009: 39: 1830-41.

26. LonG KZ, SANTOS JI. Vitamins and the regulation of the immune response. Pediatr Infect Dis J 1999: 18: 283-90.

27. Matheu V, Back O, Mondoc E, Issazadeh-Navikas S. Dual effects of vitamin D-induced alteration of $\mathrm{TH} 1 /$ TH2 cytokine expression: enhancing IgE production and decreasing airway eosinophilia in murine allergic airway disease. J Allergy Clin Immunol 2003: 112: 58592.

28. Wittke A, Weaver V, Mahon BD, August A, CAntorna MT. Vitamin D receptor-deficient mice fail to develop experimental allergic asthma. J Immunol 2004: 173: 3432-6.

29. Poon AH, Laprise C, Lemire M, et al. Association of vitamin D receptor genetic variants with susceptibility to asthma and atopy. Am J Respir Crit Care Med 2004: 170: 967-73.

30. Raby BA, Lazarus R, Silverman EK, et al. Association of vitamin $\mathrm{D}$ receptor gene polymorphisms with childhood and adult asthma. Am J Respir Crit Care Med 2004: 170: 1057-65. 